\title{
Modified cuckoo search algorithm: Feature subset selection \&Shape,Color and Texture Features Descriptors for Content-Based Image Retrieval
}

\author{
Mrs.K.Radha ${ }^{1}$, Mrs. .R.V.Sudha ${ }^{2}$, Mrs.M.Meena ${ }^{3}$, Dr.R.Jayavadivel ${ }^{4}$, S.Kanimozhi ${ }^{5}{ }^{\text {Dr.P.Prabaharan }}{ }^{6}$ \\ ${ }^{1}$ Assistant Professor, Department of computer science and engineering, Vivekanandha College of \\ Technology for women, Elayampalayam, Tiruchengode, Namakkal - Dt. \\ Mail id: radhacse.dpm@gmail.com \\ ${ }^{2}$ Assistant Professor, Department of Information Technology, Vivekanandha College of Technology for women \\ Elayampalayam, Tiruchengode, Namakkal - Dt. \\ Mail id:sudhashakthivel@gmail.com \\ ${ }^{3}$ Assistant Professor, Department of Information Technology, Vivekanandha College of Technology for women \\ Elayampalayam, Tiruchengode, Namakkal - Dt. \\ Mail id:meenasmani91@gmail.com \\ ${ }^{4}$ Associate Professor, Department of Computer Science and Engineering, \\ Vivekanandha College of Engineering for Women, Tiruchengode,Namakkal, Tamilnadu, India. 637205. E.Mail: \\ infotech.jaya@gmail.com \\ ${ }^{5}$ Assistant professor, Department of Information Technology, \\ Mkumarasamy College of Engineering, Thalavapalayam, Karur \\ E.Mail: kanimozhis.it@mkce.ac.in \\ Associate Professor, Department of computer science and engineering, Vivekanandha College of \\ Technology for women, Elayampalayam, Tiruchengode, Namakkal - Dt. \\ Mail id: kprabume@gmail.com
}

\begin{abstract}
With the recent advances in knowledge, the complication of multimedia has increased expressively and new areas of research have opened up in search of new multimedia content. Content-based image retrieval (CBIR) are used to extract images associated with image queries (IQs) from huge databases. The CBIR schemes accessible at present have limited functionality because they only have a partial number of functions. This document presents an improved cookie detection algorithm with coarse sentences for processing large amounts of data using selected examples. The improved cuckoo detection algorithm mimics the behavior of brood attachment parasites in some cuckoo species, including some birds. Modified cuckoo recognition uses approximate set theory to create a fitness function that takes into account the sum of features and the quality of classification as a small amount. For an image entered as IQ from a database, distance metrics are used to find the appropriate image. This is the central idea of CBIR. The projected CBIR method is labelled and can extract shape features based on the RGB color using the and canny Edge (CED) and neutrosophic clustering algorithm scheme. After YCbCrcolor cut, and the CED to get the features to extract the vascular matrix. The combination of these techniques improves the efficiency of the CBR image recovery infrastructure. In this thesis recursive neural network techniques are used to measure the similarity. In addition, the accuracy of the results is: The recall score is measured to evaluate system performance. The proposed CBIR system provides more precise and accurate values than the complex CBIR system.
\end{abstract}


Keyword:Modified cuckoo search algorithm, CBIR, Shape features,Metaheuristic algorithm, recursive neural network, and Similarity measure

\section{Introduction}

With recent technological advances, the complexity of multimedia has increased significantly, opening up new areas of research in search of new multimedia content. CBIR systems are used to extract IQ images from enormous databases. The CBIR schemesaccessiblenowadays have partial functionality because they have only a limited number of functions. This document presents an improved cookie detection algorithm with rough recommendations for handling large amounts of data using selected examples. The improved culling detection algorithm is similar to hatchery pest behavior in several cuckoo species, including some birds. Modified recognition for a hill uses quantitative theory approximately to create a fitness function that takings the number of functions and the quality of classification as small. For an image pass in as IQ from the database, distance metrics are used to obtain a suitable image. This is the core idea of CBIR [1-3]. The projected CBIR method is labelled and can achieve shape functions based on RGB color using CEDtechnique, YCbCr fanned with color shaving and canny Edge histogram in functions to remove vascular matrix. The combination of these techniques improves the efficiency of CBR image retrieval infrastructure [4-6]. This work uses RNN methods to measure similarity. In addition, the accuracy of the results is as follows. The recall score is measured to assess the presentation of the system. The proposed CBIR system provides more accurate and precise values than the complex CBIR system [7-9].

Several visual descriptions have been suggested in the literature. Some of these characters are common, while some apply to particularrequests [10]. However, there is a difference among the user's semantic interest and the visual cue extracted by the reporter. For example, if the user can provide a red apple image upon request, the resulting images may include red roses, red balls or green apples contingent on the pictorial appearance achieved by the CBIR system. Lately, various query search engines have emerged as a technique to overwhelmed this gap and fill in significant gaps by using low-level image functions [11]]. For these schemes, the user delivers a series of QI. This provides a better considerate of the user's high near of interest in search engines.

The rest of the work is done as shadows. In section 2, we will look at work associated to several query recovery systems. The suggested approach - "resistance learners" - is labelled in section 3. In section 4, we evaluate the effectiveness of the resistance approach. Lastly, in section 5 , we draw conclusions and outline future work.

\section{Related works}

Das et al. [12] improve image search and recognition using CBIR scheme. In this revision, the authors tested the projected system using two publicly available data sets with a total of 3688 images. Using this technique, the number of features was determined to be twelve irrespective of size. Statistical measures to appraise the proposed work. 
Ashraf et al. [13] using the bandelet transformation, with this method, info about the chiefsubstances of the image is obtained consistent. The authors used artificial neural networks to obtain images. Consequently, the authors used three publicly available datasets when evaluating the presentation and use of the projected method. Coil, and Caltech 101 datasets were used. The authors used precision and retention values to assess recovery efficiency.

Zeng [14] Introduction of the new Local Structure Descriptor (LSD) for removing color imageries. In this document, the depiction of spatial structures refers to the similarity of the edge orientation. Temporarily, primary colors from local structures are used to create LSD. LSD associations color, texture and overall size for efficient image restoration. Using a local structure histogram (LSH) to mine the vectors of the properties of every image in HVS (color, saturation and value) uses techniques to describe statistical and structural textures. Furthermore, the use of the proposed feature extraction procedure does not need that the user be trained on a large sum of training data sets. In addition, the histogram of the local structure can be extracted according to the LSD. The experiments used a database of Corel images and the results approve the superiority of the image search performance of the projected descriptor over others.

Zhou et al. [15]. In this view, the authors used an effective combination of two types of histograms drawn from local directional pattern (LDP). In implementing the projected approach, the novelists first described the extraction procedure of color and LDP histograms. Feature Two features were introduced before creating an effective merger process that included normalization and the new relationship metric. The authors associated the results of the proposed method with other pre-art approaches based on two reference datasets. The projectedattitude was therefore examined. Finally, the authors created a user-friendly for the specific recovery system.

Ali et al. [16]used a combination of Scale Invarient Feature Transform and Speed-up Robust. For refurbishmentactivities, the representation of these local characteristics is appropriate and adequate because the scale-based characteristic demonstrates the resistance to transformer rotation and scaling. In addition, robust acceleration features are more resistant to light changes. Scale up the fixed functional transducer and accelerate robust functionality while improving CBR efficiency. In this study, the authors used Coral-2000, Coral-1500 and Coral1000 for comparison and evaluation.

Feng et al. [17] has used the Global correlation (GC) for retrieval the image search. This proposed method draws texture and color properties for CBIR. In addition, the correlation values of the structural components and the histogram statistics were joined. This method provided a description of the texture and color properties. The authors used the Corel-5K and Corel-10K data sets for verification. The authors used feedback and accuracy to assess effectiveness.

\section{Proposed System}

The proposed CBIR engineering system was tested using numerous QI and related imageries obtained from database imageries. This thesis shows some of these features in a repository as a dedicated vector and finds a large number of strong and important features from an image database. Features include color signatures, sizes, and texture measurements. Here the characteristics are extracted from a specific IQ. Consequently, innovative analogies with 
metauristic algorithms [modified cuckoo search algorithms] were used to evaluate the functions of IQ and those associated with images in the database. For an image go into as an IQ from a, distance metrics are used to find the matching image. This is the major concept behind CBIR.

The projected CBIR approaches are labelled and manufactured by the same method based on the neutrosophic clustering algorithm and RBB color and are used to extract the shape properties. The YCBCR description was used to draw the color characteristics of the wave transformation color and the Virgo Edge histogram and was used to draw the characteristics of the GLCM texture. Performed Functions All generated features are stored and stored in the repository. After removing the functions from the database imageries, anevaluation was made among the entered QI and the related images in the function store. Therefore, the cuckoo search algorithm creates a random egg (pop size) with length $\mathrm{n}$. In addition, the sum of intelligencerelated images determines the number of genes in each egg. The generated egg and query image properties are removed and then these eggs are converted and the crossover is operated in addition to the local selection method and SA. This leads to the search for the best eggs. After completing crossover, transformation, and local SA search tasks, the best eggs are selected and these chromosomes are indexed into images corresponding to the query image. And to improve the level of similarity in this essay, recursive neural network technology is used. This process is repeated until the maximum repetition (Itermax 1000). Here, the M.A. Was applied. The flow of the projectedscheme is defined in Figure 1.

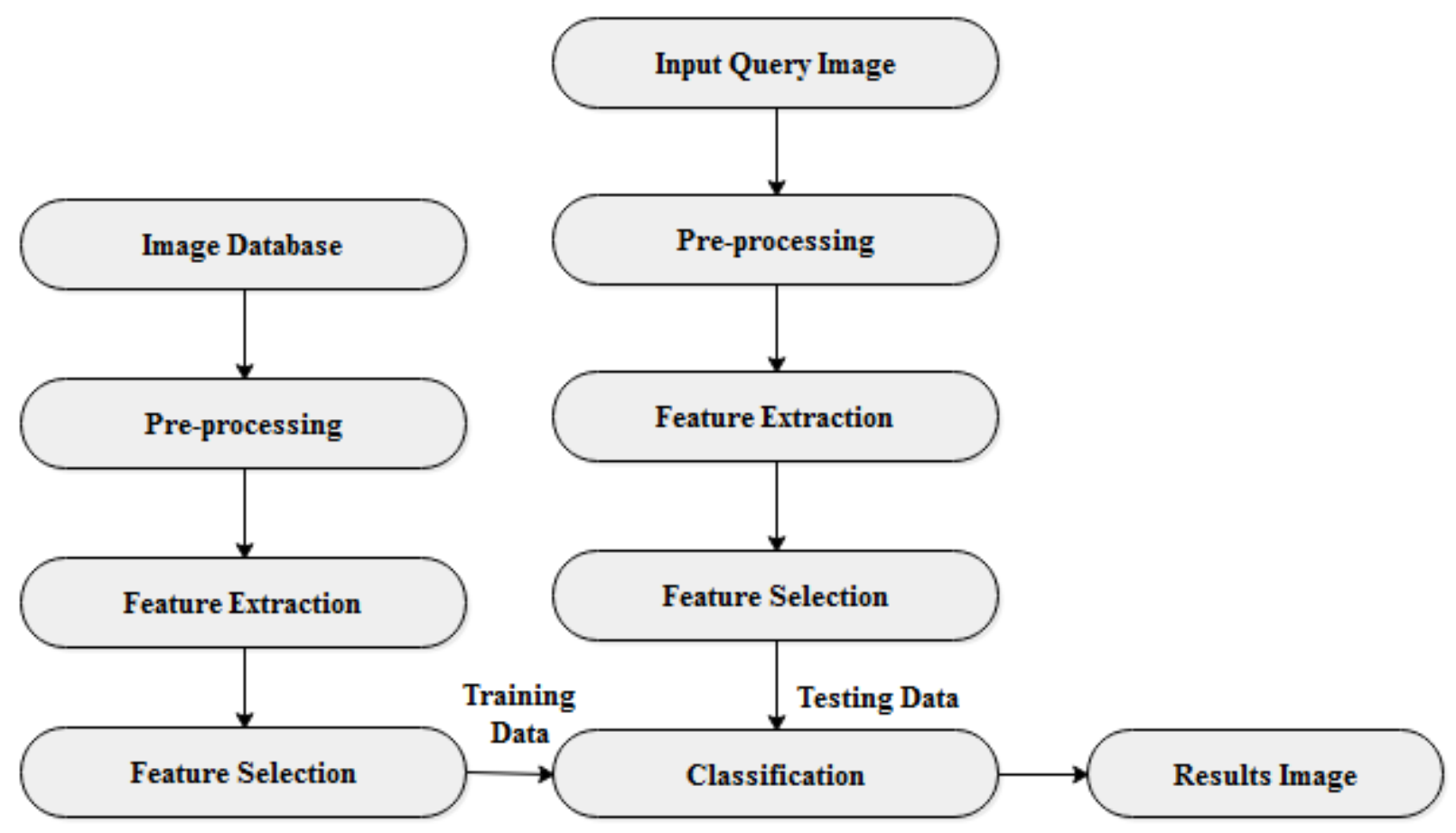

Fig.1. Proposed system

\subsection{Dataset description}


The experiments performed in this study consisted of 1000 custom images from the Corel data set (a) and each image in the data set was $384 * 256$ or $256 * 384$. By the way, the grades were shown using 10 semantic sets and each set comprises a 100imageries. Coral datasets fall into the subsequent semantic groups: mountains, Africa, houses, buses, elephants, horses, beaches and flowers. These expressive classes were used to explosion the outcomes according to a major study used these groups to demonstrate the efficiency of their CBIR approaches. Therefore, using the documented results, the results can be clearly compared. Fig. 2 shows samples of diverse images from 10 class of Corel data sets.
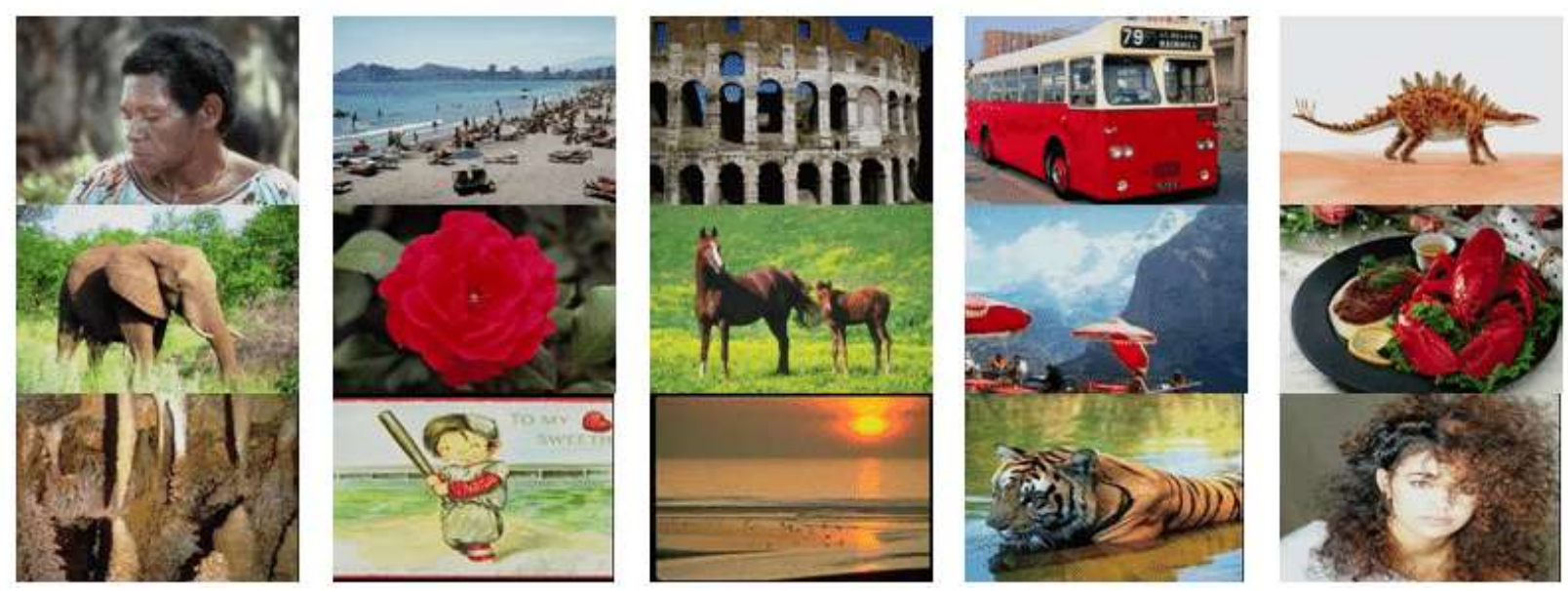

Fig.2. Corel dataset sample images.

\subsection{Pre-processing}

In this proposed system pre-processing contains with the help of three major steps such as RGB image to YCbCr conversion, Median filter and canny edge detection. The proposed Preprocessing procedures defined in the figure 3. 


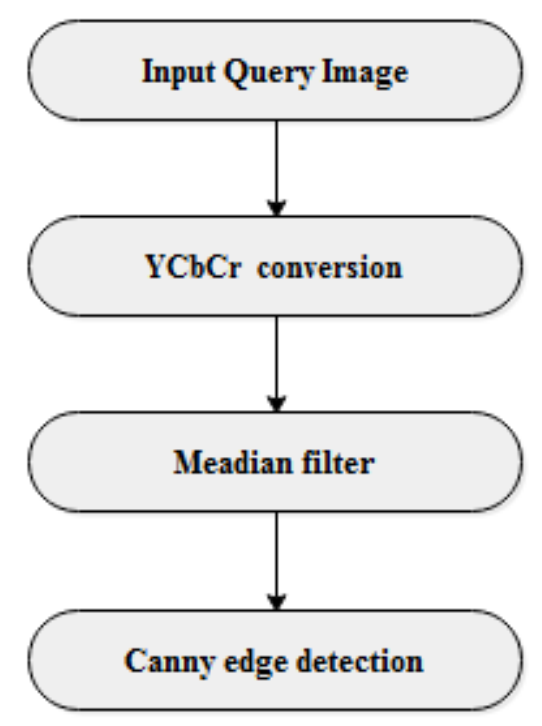

Fig.3. Proposed Pre-processing procedure.

\subsubsection{RGB image to $\mathrm{YCbCr}$ conversion}

An important function of imaging of plexuses is the conversion of color space. Live images and videos are put in storage in RGB format, because they depend on the compassion of colorseeking cells in the human visual system. $\mathrm{YCbCr}$ is widely used in color space digital imaging to take benefit of the low resolution capabilities of human optical systems for colors in terms of luminance [18]. That is why RGB to $\mathrm{YCbCr}$ is extensively used in conversion of images method.

Given the digital pixels signified in RGB format, 8 bits per example, where 0 and 255 represent respectively black and white, can be obtained from the equations of the YCBCR element.1:

$$
\left\{\begin{array}{l}
Y=16+\frac{65.738 R}{256}+\frac{129.057 G}{256}+\frac{25.064 B}{256} \\
C b=128-\frac{37.945 R}{256}-\frac{74.494 G}{256}+\frac{112.439 B}{256}(1) \\
C r=128+\frac{112.439 R}{256}-\frac{94.154 G}{256}-\frac{18.285 B}{256}
\end{array}\right.
$$

Equation 1 finding the nearest integer, multiplying and replacing the portion with a shift register yields equation 2 :

$$
\left\{\begin{array}{c}
Y=16+(((R \ll 6)+(R \ll 1)+(G \ll 7)+G+(B \ll 1)+(B \ll 3)+B) \gg 8 \\
C b=128+((-((R \ll 5)+(R \ll 2)+(R \ll 1)-((G \ll 6)+(G \ll 3)+(G \ll 1)+(B \ll 7) \gg 8) \\
C r=128+(((R \ll 7)-(R \ll 4)-((G \ll 6)+(G \ll 5)-(G \ll 1)-(B \ll 4)-(B \ll 1)) \gg 8)
\end{array}\right.
$$

The image is consuming a huge data. because that images in RGB format [19]. However, it is not worth storing or transmitting info in this color space representation, as it has a wide bandwidth. Therefore, for this, all pixels must be transformed to YCbCr.

\subsubsection{Median filter}


The medium value must actually be the value of one of the neighboring pixels, the medium filter does not create a new impractical pixel value when the filter extends to the edge. For this reason, a medium filter keeps sharp edges better than a medium filter. It is especially good to remove the impulse sound from the image. [20].

\subsubsection{Canny edge detection (CED)}

CED is the techniques of edge detection procees done in image concept and also uses multi-level algorithms to detect wide edges in images. This is John F. Established by canny in 1986. canny also developed the and explained why this technique works. The CAD algorithm consists of 5 steps such as noise reduction, gradient calculation and a detailed description of the CED [21].

\subsection{Feature Extraction}

In this proposed system, there are three different types of feature extraction process such asshape, color, and texture. Using the CBIR system, the elements are extracted from the image using various traction methods. In addition, the properties of each image from the list are stored in a discreterecord called a database function.

\subsubsection{Color FeaturesExtraction}

The combination of features, also called the combination of features, is used for better performance. This is due to the fact that one or a partialsum of functions are not resistant to important transformations (such as translation, rotation, lighting changes) occurring in the image record. This study proposes a CBIR method based on the characteristics of the elongated color. The color function is one of the most complete functions and refers to image, background and forefront objects. Color is also strong against image rotation and translation. In addition, different applications use different color characteristics. The color space defines the exact combination and display function of color models.

The resulting start and end images are displayed after the colored border is recognized. The distance factor among the feature vector of the image transferred as a query and the images from the record is used here. In a situation in which the distance among the requested image characteristic vector and the imageries from the record is minor enough, the conforming image is selected in the database as a like image. In addition, an analog index is used to indicate the distance. The examine results are then organized by the identical index.

$$
D=\sum_{I=1}^{n}\left|F_{q}-F_{r}\right|
$$

As query is signifiedby $F_{q}$, and the record feature query vector is given by $F_{r}$ in image vector feature.

\subsubsection{Shape FeaturesExtraction}

Extraction of form elements is mainly done to seizure the shape properties (such as moment, area, border, etc.) of the appearance elements. This transcript facilitates storage, broadcast and proof of identity of forms. It is very important that the form elements are resistant 
to rotation, movement and scaling. Research has been done to find out how to best find shape features to identify memory, transmission or shape. As a result, the selected form functions do not use mathematical conversion. For every pixel, a color image has color value of RGB color is transformed to grayscale to extract image size characteristics. It uses the formula suggested by Craig as shown below:

$$
I_{g}=\left[I_{r} I_{g} I_{b}\right] *[0.29890 .114](4)
$$

Based on 4 th equation, $I_{g}$ denotes the joint $2 \mathrm{D}$ matrix; $I_{r} I_{g} I_{b}$ signifies the components of colored image; $I_{g}$ is signified as the gray-level joint image. The preprocessing stage uses a medium filter to reduce the noise. Using middle filters will reduce the noise of sparks, pepper and salt. In addition, the middle filter can sharp edges in digital imageries. If edge blurring is undesirable, it is applied. Using media filters will reduce almost all of the audio in the image. Consequently, the neutrosophic clustering procedure is then used to discrete pixels with very narrow values and discount intact pixels from the gray imageries.

The last task after CED method. Boundaries around the same pixel must be defined. In this work, the CED was used to retrieve the form elements. After applying the complex edge detection method, you can get the various shapes present in the $I_{g}$ image. These markers are stored in the database using the vector form validation method. $I_{g}$ images can have different existing sizes. After the generated content index information. These index characteristics are stored in the database as vectors.

\subsubsection{Texture FeaturesExtraction}

In image classification, the GLCM seems to be a reliable and efficient technique that performs statistical analysis of images and can be interpreted as a matrix with 2-D of cumulative similarity amongsets of pixels arranged in certain directions between them. 14 tags have now been removed to classify plot features. In a sense, these acquired traits appear to be extremely correlated. To avoid similar problems that arise in this work, five structures are used for association. Therefore, the next steps are to extract the texture features using the GLCM:

Table.1. The texture features extraction using GLCM.

1. Converting the color image into a grayscale image.

2. The input image is filtered by the $5 \times 5$ Gaussian filter.

3. The filtered image is split into blocks of $4 \times 4$.

4. GLCM computes energy, standard deviation, mean value, homogeneity and contrast for each block. Computation is made to these features in accordance with four directions namely diagonal $\left(45^{\circ}\right.$ and $\left.135^{\circ}\right)$, vertical $\left(0^{\circ}\right)$ and horizontal $\left(90^{\circ}\right)$ directions.

5. The extracted features are stored in the database.

\subsection{Feature selection}


This section presents the MCS algorithm with two variations. According to [2], the first change is that the increase in the number of generations (due to inertia in the PSO) decreases.

A starting value $\alpha=b=1$ and a new value $\alpha=B / \sqrt{G}$, were chosen, which was chosen, generation being the number and B the limit. In contrast to SC, where where is constant and normally the value $=1$. The solution (eggs) is sorted according to the fitness order $f\left(x_{i}\right)$ and contained in the best, the eggs are considered elite. Second, the cuckoo is represented as a crossover: (1) For all early elite eggs $x_{i}$, arbitrarily select $x_{j}$ from elite eggs. (2) A novel solution (egg) $x_{k}$ is created by calculating the distance between them using the solution $x_{i}$ and $x_{j}$, There are two conducts to calculate the distance: the chief is the average, where if $f\left(x_{i}\right)=f\left(x_{j}\right)$, the distance is $d x=\left|x_{i}-x_{i}\right| / 2$. The another method used the reverse golden ratio $\varphi=(1+\sqrt{5}) / 2$, where $\mathrm{d} d x=\left|x_{i}-x_{i}\right| / \varphi$ if $\left|x_{i}-x_{i}\right|$ (That is, identical eggs rand $\alpha=\frac{B}{G^{2}}$. (3). were taken twice from randomly selected nests. A random nest is designated from all nest sets and a novelsolution $f\left(x_{1}\right)$, then $x_{l}=x_{k}$, is associated with the solution.

The hybrid feature selection algorithms presented in this article are shown in Figure 4. The fitness feature is evaluated in a parallel environment to minimize the evaluation. We display the facts of the MCSRS procedure below.

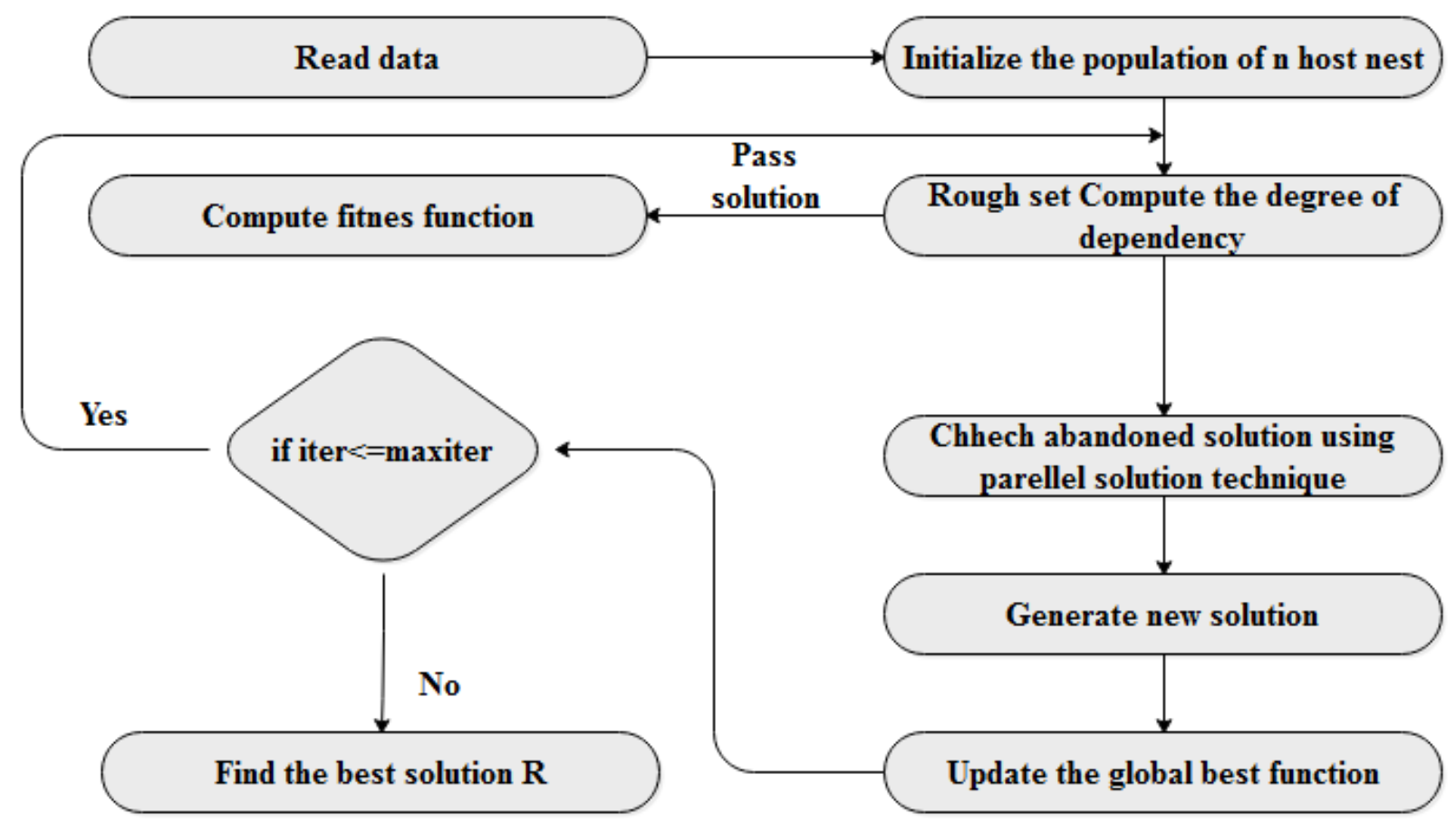

Fig.4. Flowchart of proposed Feature selection algorithm.

\subsubsection{Position Representation}

Traditional MCS positions are represented by a constant value position. Conversely, in binary MCS, the feature selection state is indicated by a logical vector in which the 
corresponding 1 functions are selected instead of the corresponding 0. The MCSRS procedure starts by choosing a random location for each slot. When a population is created, the point of each nest is changed by a binary vector of length $n$ (entiresum of objects):

$$
\begin{array}{r}
S\left(x_{i}^{j}(t)\right)=\frac{1}{1+e^{-x_{i}^{j}}(t)}(5) \\
x_{i}^{j}(t+1)=\left\{\begin{array}{cc}
1 & \text { if } S\left(x_{i}^{j}(t)\right)>\sigma \\
0 & \text { otherwish }
\end{array}\right.
\end{array}
$$

Where $\sigma \sim N(0,1)$ and $x_{j}$ tsignifies a novel solution at time stept. To calculate the distance between $x_{j}$ tand $x_{i} t$ the used as hamming.

\subsubsection{Fitness function scheme}

Dependent on the idea of MS, the fitness function is determined based on two influences: the sum of functions at reduced speed and the quality of the classification:

$$
F(R)=\eta \times \gamma_{c}(D)+\xi \times\left(1-\frac{\|R\|}{|C|}\right)(7)
$$

Where $\gamma_{c}(D)$ is distinct in [21] and $\mathrm{R}$ signifies the sum of functions chosen by $\mathrm{C}$ and $|\mathrm{C}|-$ the overallsum of structures in the unique set. $\xi$ and $\eta(\xi+\eta=1, \eta \in[0,1])$ are two factors. For each cuckoo, the fitness function is calculated in (7) and associated with the best overall fitness $F_{b e s t}$. If the current value $\left(F_{i}\right)$ is improved, the overall best fit is swapped by it, and its $\operatorname{location}\left(x_{i}\right)$ develops a decreasing set of $R=x_{\text {best }}$ (conforming to the best subset of functionality).

\subsection{Classification}

In this proposed system for CBIR classification recursive neural network is used the network architecture is defined in the figure.5.

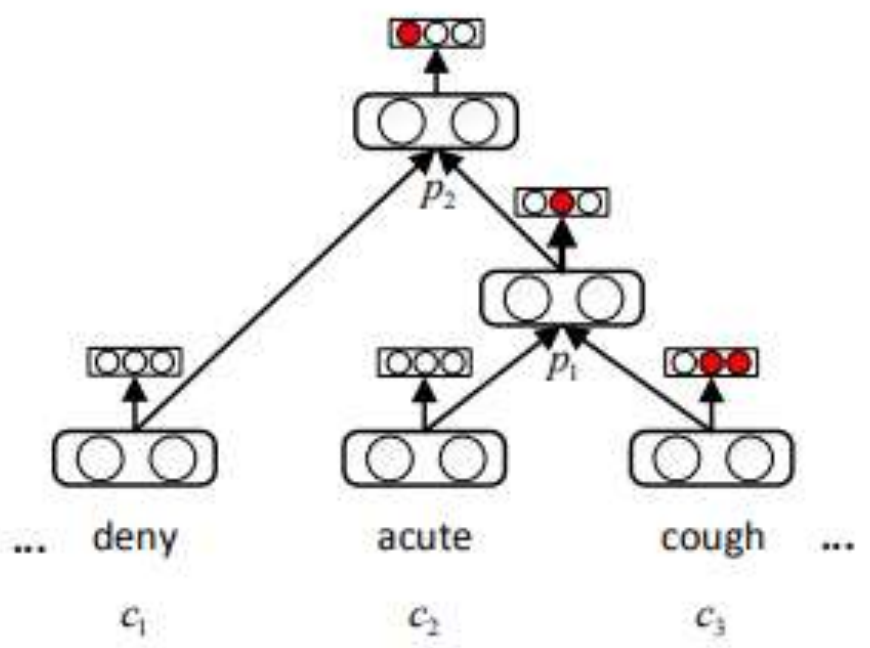

Fig.5.classification task ofRNN models. 
Standard RNNs are used as a classic neural network structure to solve complex symbolic problems for complex symbolic structures of any shape (e.g. logical terms, plants or graphs). Fig. This explains the approach. When a sentence is delivered, the RNN parses it into a binary semantic tree and calculates the vector representation of each word. During the feedforward training period, the RNN calculates the parent vector in ascending order. The structural equation looks like this:

$$
p_{1}=f\left(W\left[\begin{array}{l}
c_{2} \\
c_{z}
\end{array}\right]+b\right), p_{2}=f\left(W\left(\left[\begin{array}{l}
c_{1} \\
p_{1}
\end{array}\right]+b\right)\right.
$$

Where $f$ is the activation function; $W \in \mathbb{R}^{d \times 2 d}$ is the weight matrix, where $\mathrm{d}$ is the dimensionality of the vector; and bias is identified as the $\mathrm{b}$. Then, each parent vector $p_{i}$ is given as a feature to a softmax classifier such as that distinct in Eq. 9 to calculate its label probabilities:

$$
y^{p}=\operatorname{softmax}\left(W_{s^{*}} \cdot p\right)
$$

Where Ws $\in \mathbb{R}^{3 \times d}$ is the classification matrix. In this recursive process, the vector and classifying result of the node willgradually converge. After the vector of the leaf node is given, the RNN can ultimately map the semantic demonstrationof the complete tree into the root vector.

\section{Results and Discussion}

The projected system has been verified on Matlab (version 2018A) with a $3.0 \mathrm{GHz}$ Intel i3 processor, 1TB hard drive and 8GB RAM. To determine the efficiency of a designed system, it is compared to existing systems in the dataset. The trials in this study used a Corel (A) dataset containing 1000 different images. The Corel dataset has 10 different categories, which are labeled in table section. Some of the suggested survey outcomes are exposed in Table 2.

Table.2. Effects of CBIR by inputting image as QI.

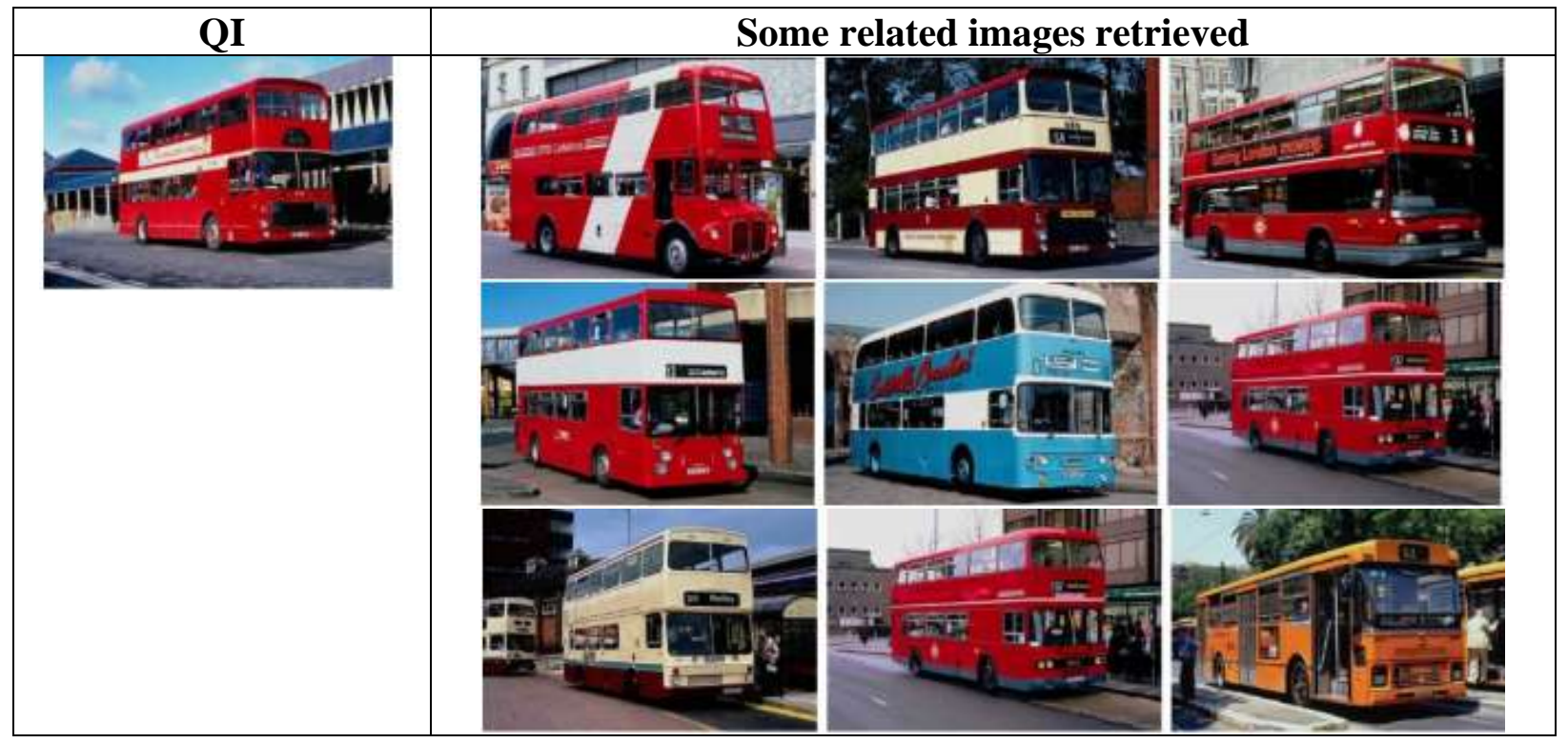




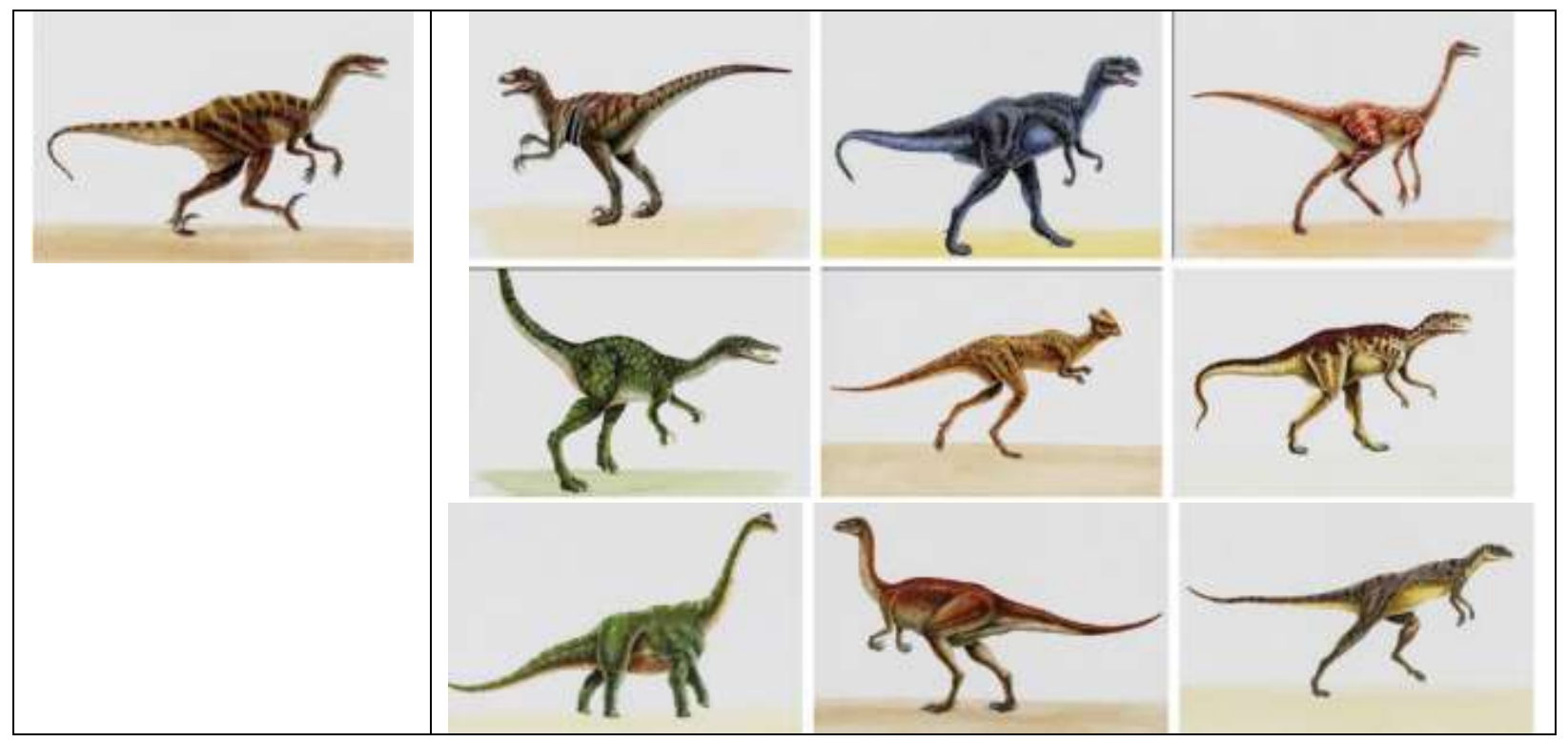

\subsection{Evaluation Metrics}

Accuracy and recall parameter are used to assess the efficiency and efficiency of the projected CBIR system. Accuracy is a degree of the aptitude of the CBIR system to retrieve a requirement-like image. In this context, the recall is a really positive coefficient and is used to assess the performance of the CBIR system in terms of the sum of related imageries restored associated to all of the same images in the database. It can be distinct as the equation for precision and speed of the recall:

$$
\begin{gathered}
\text { Precision }=\frac{\text { No.of relevent image retrived }}{\text { Total No.of image retrived }} \\
\text { Recall }=\frac{\text { No.of relevant image retrived }}{\text { Total No.of relevent images }}
\end{gathered}
$$

The outcomes of our experiment were reported by running the imitation five times. In each testing, 20 QI in each literal class were randomly designated and accuracy and recall rates were given based on the maximum 20 images.

\subsection{Performance analysis}

As seen in Table 3, the exact and recall percentages in addition to the images recovered are calculated for 20 QI. As exposed in Table 3, the projectedscheme has a high efficiency in image recovery obtaining high precision and recall values. Experiments have shown that increasing the recovery of similar images improves clarity and recall rates. The characteristics of color, shape and texture combined with the selection of the proposed characteristics have proved to be a very encouraging improvement in the whole CBIR process, especially in terms of efficiency and accuracy.

Table.3. Performance analysis of Precision and recall degrees. 


\begin{tabular}{|c|c|c|}
\hline Class & Precision & Recall \\
\hline Buses & 100 & 20 \\
\hline Mountins & 85.58 & 17.64 \\
\hline Beach & 84.65 & 17.44 \\
\hline Elephants & 91.45 & 17 \\
\hline Food & 93.77 & 16 \\
\hline Flowers & 95.87 & 18 \\
\hline Africa & 84.11 & 19 \\
\hline Horses & 94.13 & 18.44 \\
\hline Dinosaurs & 100 & 20 \\
\hline Buildings & 84.55 & 16 \\
\hline
\end{tabular}

Here, the precision and recall value is estimated by using the given dataset, which having the ten different classes. The each class in the dataset value is calculated separately. In highest precision value in bus class and dinosaurs class. And also recall value is better in every classes.

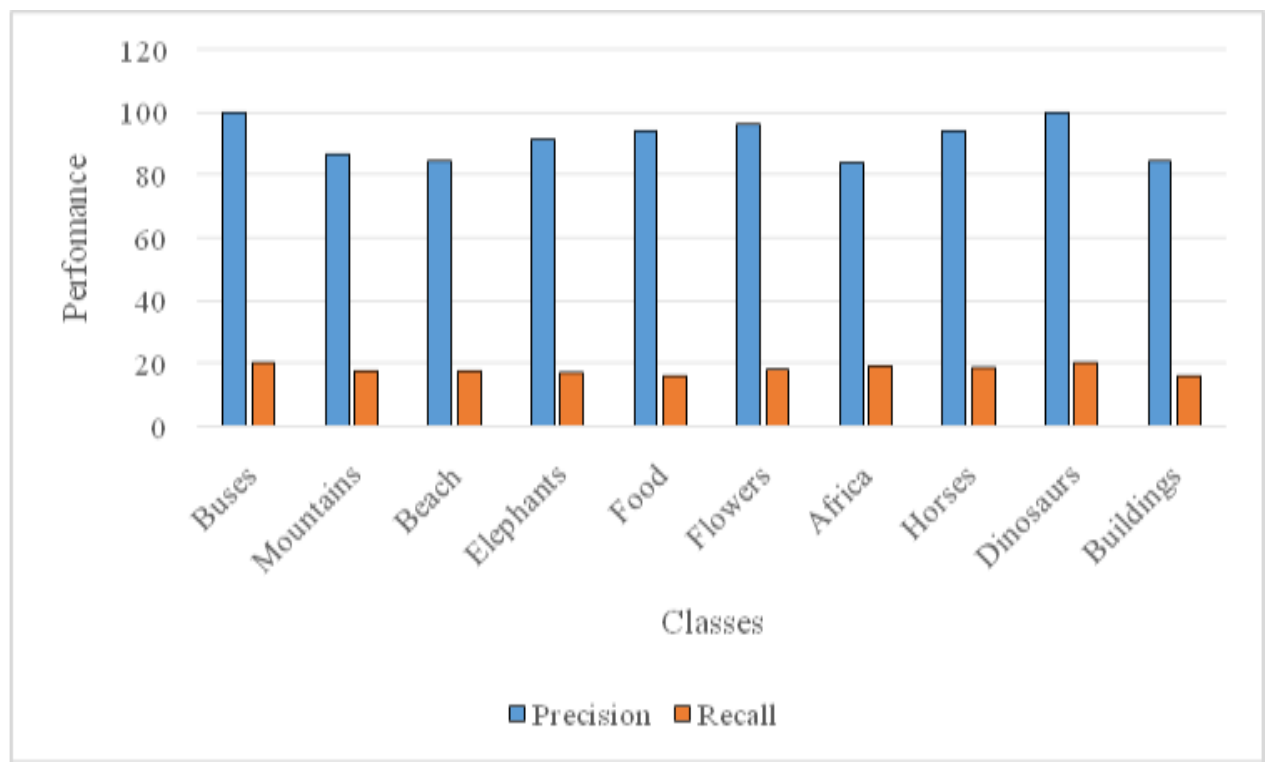

Fig.6.Performance analysis of Precision and recall rates.

\section{Comparative analysis}

To demonstrate the projectedschemeeffectiveness in terms of research capacity, the results gained are also compared with other existingschemes in terms of accuracy and recall speed. The other comparative schemes are Mehmood et al. [23], Irtaza et al. [22], Ali et al. [24] and Mehmood et al. [25] and the same ten semantic sets were used by these comparative systems.

Table.4.Comparisonof recall standards between existing systems and projected scheme. 


\begin{tabular}{|c|c|l|l|l|l|}
\hline Class & Proposed & GCCL[22] & What [23] & $\begin{array}{l}\text { Spatical } \\
\text { leve-2 [24] }\end{array}$ & $\begin{array}{l}\text { Spactial } \\
\text { LGH [25] }\end{array}$ \\
\hline Buses & 20 & 0.2 & 19.15 & 19.5 & 19.17 \\
\hline Mountains & 17.64 & 0.14 & 17.15 & 16.57 & 16.49 \\
\hline Beach & 17.44 & 0.14 & 15.91 & 14.44 & 14.92 \\
\hline Elephants & 17 & 0.16 & 17.91 & 18 & 17.53 \\
\hline Food & 16 & 0.18 & 16.98 & 16.54 & 15.79 \\
\hline Flowers & 18 & 0.17 & 17.37 & 18.8 & 17.03 \\
\hline Africa & 19 & 0.17 & 15.56 & 13.58 & 14.61 \\
\hline Horses & 18.44 & 0.16 & 17.88 & 17.28 & 17.26 \\
\hline Dinosaurs & 20 & 0.19 & 19.62 & 20 & 19.59 \\
\hline Buildings & 16 & 0.17 & 16.15 & 16.97 & 16.05 \\
\hline Avg & 17.94 & 0.17 & 17.37 & 17.19 & 16.84 \\
\hline
\end{tabular}

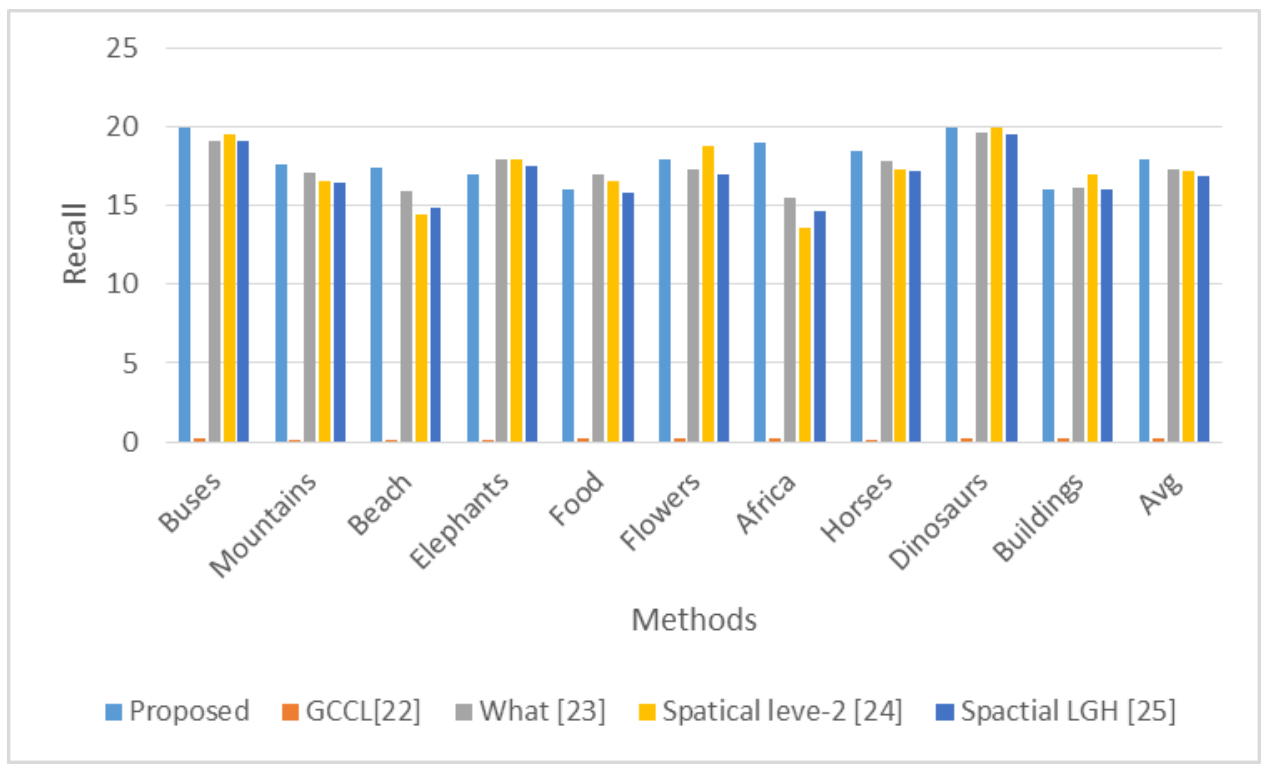

Fig.7. Comparison of average recall values between the projected and other existing schemes

In table 4 and figure 7 represent the standard recall value of both existing methods with proposed scheme. In this comparative calculation, four existing approach calculation is compared with proposed model. Bus and dinosaurs class having $20 \%$ of recall value, but the existing scheme every classes recall values are below 20. So that the proposed scheme achieve better recall value than existing methods.

\begin{tabular}{|l|c|l|l|l|l|}
\hline Class & Proposed & GCCL [22] & What [23] & $\begin{array}{l}\text { Spatical } \\
\text { level 2 [24] }\end{array}$ & $\begin{array}{l}\text { Spactial } \\
\text { LGH [25] }\end{array}$ \\
\hline Buses & 100 & 100 & 95.74 & 95.75 & 95.84 \\
\hline
\end{tabular}




\begin{tabular}{|c|l|l|l|l|l|}
\hline Mountains & 86.58 & 69 & 85.78 & 82.85 & 82.43 \\
\hline Beach & 84.65 & 72 & 79.56 & 72.2 & 74.58 \\
\hline Elephants & 91.45 & 82 & 89.54 & 89.99 & 87.64 \\
\hline Food & 93.77 & 90 & 86.87 & 85.88 & 78.96 \\
\hline Flowers & 95.87 & 86 & 77.82 & 94.01 & 85.13 \\
\hline Africa & 84.11 & 83 & 89.41 & 69.08 & 73.03 \\
\hline Horses & 94.13 & 82 & 98.12 & 86.38 & 86.29 \\
\hline Dinosaurs & 100 & 97 & 80.75 & 100 & 97.95 \\
\hline Buildings & 84.55 & 86 & 86.85 & 84.85 & 80.24 \\
\hline Avg & 91.32 & 84.70 & 86.85 & 86.10 & 84.21 \\
\hline
\end{tabular}

Table.4.Average precision values comparison between the proposed method and other existingschemes.

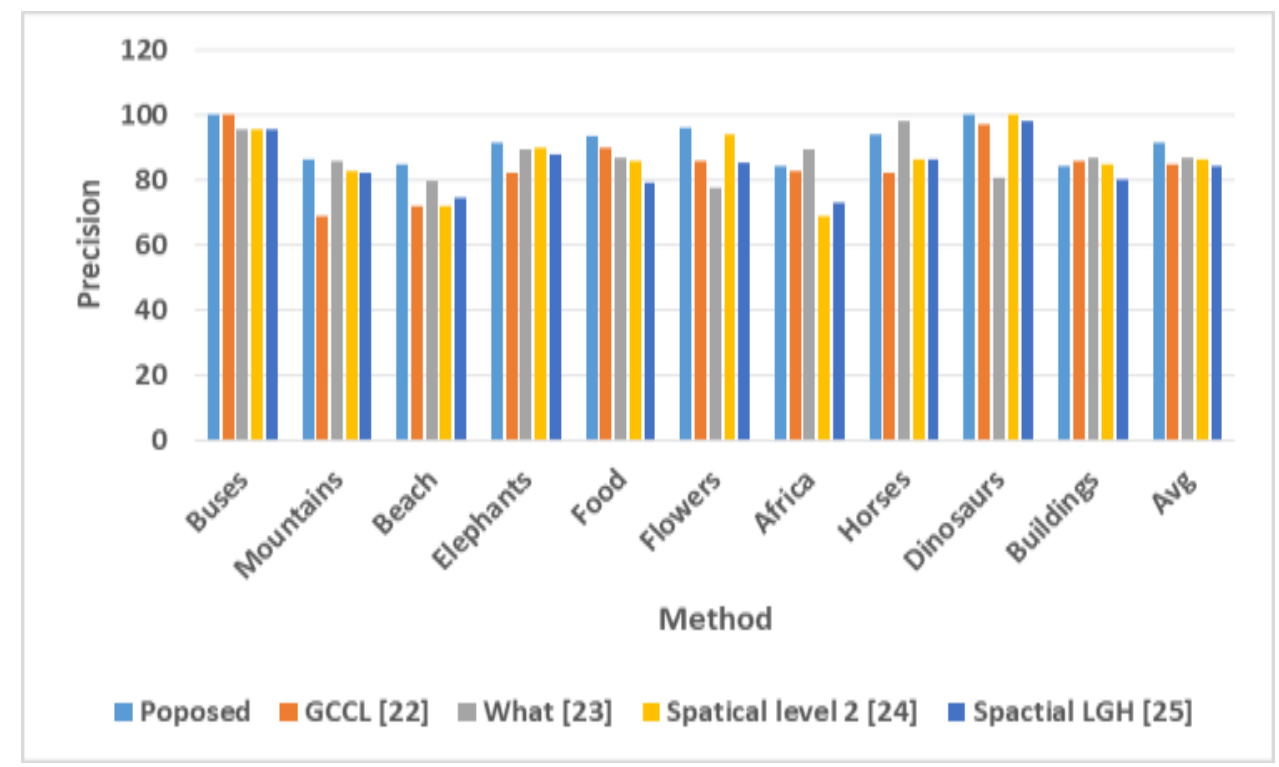

Fig.8. comparison of average precision values between the proposed scheme and other existingmethods.

In above table 4 and figure 8 signifies the average precision value of 10 classes both as proposed and existing scheme. In this comparative analysis of precision value is obtained by using corel dataset. In proposed method attained $100 \%$ precision value in bus class. And the 95.87\% attained in flower class. But the existing scheme attained precision value below 97\%, but [24] scheme achieved 100\% precision value in Dinosaurs class. Also [24] method attained 100\% precision value in bus class. But this methods achieve another classes precision value is slightly lower than proposed method.

\section{Conclusion}


This study proposed an efficient CBIR system that MA uses to restore images from a database. Once the user enters a request image, the image features are removed from the image using the projected CBIR system. These drawn elements include shape, color, and texture. Specifically, a YCBR color histogram and a CED histogram with various wavelet converters were used to extract color properties, RGB color and Kenny Edge's method with neutrophysical clustering algorithms were used, and the size and properties of the GLCM were extracted. This was used to extract plot features. Subsequently, images corresponding to the query image were extracted efficiently using similarity measurements based on metaturistic algorithms. The research was carried out using the Corel data set and the projected similarity measures based on the metaturistic algorithm showed a high ability to differentiate between color, shape and texture features. In this work, the recursive neural network method is used to measure the similarity, which improves the proposed efficiency. In our proposed system performs better than traditionlapproaches and has shown promising results in image recovery in terms of accuracy and recall in numerouscollections of Corel image records, and the proposed system exhibits excellent overall results in terms of accuracy and recall when equated to others existing systems.

\section{Reference}

[1]. Faloutsos, C.; Barber, R.; Flickner, M.; Hafner, J.; Niblack, W.; Petkovic, D.; Equitz, W. Efficient and effective querying by image content. J. Intell. Inf. Syst. 1994, 3, 231-262.

[2]. Flickner, M.; Sawhney, H.; Niblack, W.; Ashley, J.; Huang, Q.; Dom, B.; Gorkani, M.; Hafner, J.; Lee, D.; Petkovic, D.; et al. Query by image and video content: The QBIC system. Computer 1994, 28, 23-32.

[3]. Nepal, S.; Ramakrishna, M. A generalized test bed for. In Proceedings of the 10th International Conference of the Information Resources Management Association, Hershey, PA, USA, 16-19 May 1999.

[4]. Jiang, M.; Zhang, S.; Li, H.; Metaxas, D.N. Computer-Aided Diagnosis of Mammographic Masses Using Scalable Image Retrieval. IEEE Trans. Biomed. Eng. 2014, 62, 783-792.

[5]. Zhang, X.; Liu, W.; Dundar, M.; Badve, S.; Zhang, S. Towards Large-Scale Histopathological Image Analysis: Hashing-Based Image Retrieval. IEEE Trans. Med. Imaging 2014, 34, 496-506.

[6]. Alraqibah, H.; Bchir, O.; Ismail, M.M.B. X-ray Image Retrieval System Based on Visual Feature Discrimination. In Proceedings of the International Conference on Digital Image Processing (ICDIP), Athens, Greece, 5-6 April 2014.

[7]. Guo, J.M.; Prasetyo, H.; Chen, J.H. Content-Based Image Retrieval Using Error Diffusion Block Truncation Coding Features. IEEE Trans. Circ. Syst. Video Technol. 2014, 25, 466481.

[8]. Thepade, S.D.; Shinde, Y.D. Robust CBIR using sectorisation of hybrid wavelet transforms with Cosine-Walsh, Cosine-Kekre, Cosine-Hartley combinations. In Proceedings of the International Conference on Pervasive Computing (ICPC), Pune, India, 8-10 January 2015.

[9]. Gupta, N.; Das, S.; Chakraborti, S. Extracting information from a query image, for content based image retrieval. In Proceedings of the Eighth International Conference on Advances in Pattern Recognition (ICAPR), Kolkata, India, 4-7 January 2015. 
[10]. Otaiba, S.; Qassim, S.; Bchir, O.; Ismail, M.M.B. Empirical comparison of visual descriptors for multiple bleeding spots recognition in wireless capsule endoscopy video. In Proceedings of the International Conference on Computer Analysis of Images and Patterns (CAIP), York, UK, 27-29 August 2013.

[11]. Hsiao, K.J.; Calder, J.; Hero, A.O. Pareto-Depth for Multiple-Query Image Retrieval. IEEE Trans. Image Process. 2014, 24, 583-594.

[12]. Das, R.; Thepade, S.; Bhattacharya, S.; Ghosh, S.: Retrieval architecture with classified query for content based image recognition. Appl. Comput. Intell. Soft Comput. 2016, 2 (2016).

[13]. Ashraf, R.; Bashir, K.; Irtaza, A.; Mahmood, M.T.: Content based image retrieval using embedded neural networks with bandletized regions. Entropy 17(6), 3552-3580 (2015).

[14]. Zeng, Z.: A novel local structure descriptor for color image retrieval. Information 7(1), 9 (2016).

[15]. Zhou, J.-X.; Liu, X.-D.; Xu, T.-W.; Gan, J.-H.; Liu, W.-Q.: A new fusion approach for content based image retrieval with color histogram and local directional pattern. Int. J. Mach. Learn. Cybern. 9(4), 677-689 (2018).

[16]. Ali, N.; Bajwa, K.B.; Sablatnig, R.; Chatzichristofis, S.A.; Iqbal, Z.; Rashid, M.; Habib, H.A.: A novel image retrieval based on visual words integration of SIFT and SURF. PLoS ONE 11(6), e0157428 (2016).

[17]. Feng, L.; Wu, J.; Liu, S.; Zhang, H.: Global correlation descriptor: a novel image representation for image retrieval. J. Vis. Commun. Image Represent. 33, 104-114 (2015).

[18]. Walton S, Hassan O, Morgan K, Brown MR (2011) Modified cuckoo search: a new gradient free optimization algorithm. Chaos Solitons Fractals 44(9):710-718.

[19]. Yang X-S, Deb S (2010) Engineering optimization by cuckoo search. Int J Math Model NumerOptim 1:330-430.

[20]. Leondes, Cornelius T. Multidimensional Systems: Signal Processing and Modeling Techniques: Advances in Theory and Applications. Elsevier, 1995.

[21]. Ma, Xiaoju, Bo Li, Ying Zhang, and Ming Yan. "The Canny edge detection and its improvement." In International Conference on Artificial Intelligence and Computational Intelligence, pp. 50-58. Springer, Berlin, Heidelberg, 2012.

[22]. Irtaza, A.; Adnan, S.M.; Ahmed, K.T.; Jaffar, A.; Khan, A.; Javed, A.; Mahmood, M.T.: An ensemble based evolutionary approach to the class imbalance problem with applications in CBIR. Appl. Sci. 8(4), 495 (2018).

[23]. Mehmood, Z.; Mahmood, T.; Javid, M.A.: Content-based image retrieval and semantic automatic image annotation based on the weighted average of triangular histograms using support vector machine. Appl. Intell. 48(1), 166-181 (2018).

[24]. Ali, N.; Bajwa, K.B.; Sablatnig, R.; Mehmood, Z.: Image retrieval by addition of spatial information based on histograms of triangular regions. Comput. Electr. Eng. 54, 539-550 (2016).

[25]. Mehmood, Z., Anwar, S.M., Ali, N., Habib, H.A., Rashid, M.A.: novel image retrieval based on a combination of local and global histograms of visual words. Math. Probl. Eng. 2016, 1-12 (2016). 